\title{
Edge Colorings of Planar Graphs without 6-Cycles with Two Chords*
}

\author{
Ling Xue ${ }^{1}$, Jianliang $\mathrm{Wu}^{2}$ \\ ${ }^{1}$ Department of Information Engineering, Taishan Polytechnic, Tai'an, China \\ ${ }^{2}$ School of Mathematics, Shandong University, Jinan, China \\ Email: jlwu@sdu.edu.cn
}

Received January 8, 2013; revised March 20, 2013; accepted April 18, 2013

Copyright (C) 2013 Ling Xue, Jianliang Wu. This is an open access article distributed under the Creative Commons Attribution License, which permits unrestricted use, distribution, and reproduction in any medium, provided the original work is properly cited.

\begin{abstract}
It is proved here that if a planar graph has maximum degree at least 6 and any 6 -cycle contains at most one chord, then it is of class 1 .
\end{abstract}

Keywords: Edge Coloring; Planar Graph; Cycle; Class 1

\section{Introduction}

All graphs considered here are finite and simple. Let $\mathrm{G}$ be a graph with the vertex set $V(G)$ and edge set $E(G)$. If $v \in V(G)$, then its neighbor set $N_{G}(v)$ (or simply $N(v))$ is the set of the vertices in $G$ adjacent to $v$ and the degree $d(v)$ of $v$ is $\left|N_{G}(v)\right|$. We denote the maximum degree of $G$ by $\Delta(G)$. For $V^{\prime} \subseteq V(G)$, we denote $N\left(V^{\prime}\right)=\bigcup_{u \in V^{\prime}} N(u)$. A $k^{-}, k^{+}$-vertex is a vertex of degree $k$, at least $k$. A $k$ (or $k^{+}$)-vertex adjacent to a vertex $x$ is called a $k\left(\right.$ or $\left.k^{+}\right)$-neighbor of $x$. Let $d_{k}(x)$, $d_{k+}(x)$ denote the number of $k$-neighbors, $k^{+}$-neighbors of $x$. A $k$-cycle is a cycle of length $k$. Two cycles sharing a common edge are said to be adjacent. Given a cycle $C$ of length $k$ in $G$, an edge

$x y \in E(G) \backslash E(C)$ is called a chord of $C$ if

$x, y \in V(C)$. Such a cycle $C$ is also called a chordal$k$-cycle.

A graph is k-edge-colorable, if its edges can be colored with $k$ colors in such a way that adjacent edges receive different colors. The edge chromatic number of a graph $G$, denoted by $\chi^{\prime}(G)$, is the smallest integer $k$ such that $G$ is $k$-edge-colorable. In 1964, Vizing showed that for every simple graph $G, \Delta(G) \leq \chi^{\prime}(G) \leq \Delta(G)+1$. A graph $G$ is said to be of class 1 if $\chi^{\prime}(G)=\Delta(G)$, and of class 2 if $\chi^{\prime}(G)=\Delta(G)+1$. A graph $G$ is critical if it is connected and of class 2 and $\chi^{\prime}(G-e)<\chi^{\prime}(G)$ for any edge $e$ of $G$. A critical graph with maximum degree $\Delta$ is called a $\Delta$-critical graph. It is clear that every critical graph is 2-connected.

${ }^{*}$ This work was partially supported by NNSF of China (No. 11271006).
For planar graphs, more is known. As noted by Vizing [1], if $C_{4}, K_{4}$, the octahedron, and the icosahedron have one edge subdivided each, class 2 planar graphs are produced for $\Delta \in\{2,3,4,5\}$. He proved that every planar graph with $\Delta \geq 8$ is of class 1 (There are more general results, see [2] and [3]) and then conjectured that every planar graph with maximum degree 6 or 7 is of class 1 . The case $\Delta=7$ for the conjecture has been verified by Zhang [4] and, independently, by Sanders and Zhao [5]. The case $\Delta=6$ remains open, but some partial results are obtained. Theorem 16.3 in [1] stated that a planar graph with the maximum degree $\Delta$ and the girth $g$ is of class 1 if $\Delta \geq 3$ and $g \geq 8$, or $\Delta \geq 4$ and $g \geq 5$, or $\Delta \geq 5$ and $g \geq 4$. Lam, Liu, Shiu and $\mathrm{Wu}$ [6] proved that a planar graph $G$ is of class 1 if $\Delta \geq 6$ and no two 3-cycles of $G$ sharing a common vertex. Zhou [7] obtained that every planar graph with $\Delta \geq 6$ and without 4 or 5 -cycles is of class $1 . \mathrm{Bu}$ and Wang [8] proved that every planar graph with $\Delta \geq 6$ and without 6-cycles is of class 1 . Ni [9] extended the result that every planar graph with $\Delta \geq 6$ and without chordal 6 -cycles is of class 1 . In the note, we improve the above result by proving that every planar graph with $\Delta \geq 6$ and without 6-cycles with two chords is of class 1 .

\section{The Main Result and Its Proof}

To prove our result, we will introduce some known lemmas.

Lemma 1. (Vizing's Adjacency Lemma [1]). Let $G$ be a $\Delta$-critical graph, and let $u$ and $v$ be adjacent vertices of $G$ with $d(v)=k$. 
1) If $k<\Delta$, then $u$ is adjacent to at least $\Delta-k+1$ vertices of degree $\Delta$;

2) If $k=\Delta$, then $u$ is adjacent to at least two vertices of degree $\Delta$.

From the Vizing's Adjacency Lemma, it is easy to get the following corollary.

Corollary 2. Let $G$ be a $\Delta$-critical graph. Then

1) Every vertex is adjacent to at most one 2-vertex and at least two $\Delta$-vertices;

2) The sum of the degree of any two adjacent vertices is at least $\Delta+2$;

3) If $u v \in E(G)$ and $d(u)+d(v)=\Delta+2$, then every vertex of $N(\{u, v\}) \backslash\{u, v\}$ is a $\Delta$-vertex.

Lemma 3 [4]. Let $G$ be a $\Delta$-critical graph, $u v \in E(G)$ and $d(u)+d(v)=\Delta+2$. Then

1) every vertex of $N(N(\{u, v\})) \backslash\{u, v\}$ is of degree at least $\Delta-1$;

2) if $d(u), d(v)<\Delta$, then every vertex of

$N(N(\{u, v\})) \backslash\{u, v\}$ is a $\Delta$-vertex.

Lemma 4 [5]. No $\Delta$-critical graph has distinct vertices $x, y, z$ such that $x$ is adjacent to $y$ and $z$, $d(z)<2 \Delta-d(x)-d(y)+2$ and $x z$ is in at least $d(x)+d(y)-\Delta-2$ triangles not containing $y$.

To be convenient, for a plane graph $G$, let $F(G)$ be the face set of $G$. A face of a graph is said to be incident with all edges and vertices in its boundary. Two faces sharing an edge $e$ are said to be adjacent at $e$. A degree of a face $f$, denoted by $d_{G}(f)$ is the number of edges incident with $f$ where each cut edge is counted twice. A $k^{-}, k^{+}$-face is a face of degree $k$, at least $k$. A $k$-face of $G$ is denoted by $\left[v_{1}, v_{2}, \cdots, v_{k}\right]$ if it is incident with $v_{1}, v_{2}, \cdots, v_{k}$ along its boundary. A 3 -face $[x, y, z]$ of $G$ is called an $(i, j, k)$-face if

$d(x)=i \leq d(y)=j \leq d(z)=k$. For a vertex $x \in V(G)$, we denote by $f_{k}(v)$ the number of $k$-faces incident with $v$.

Lemma 5 [4,5]. If $G$ is a planar graph with $\Delta(G) \geq 7$, then $G$ is of class 1 .

Lemma 6 [8]. If $G$ is a graph of class 2, then $G$ contains a $k$-critical subgraph for each $k$ satisfying $2 \leq k \leq \Delta(G)$.

Theorem 7. Let $G$ be a planar graph with $\Delta \geq 6$. If any 6-cycle contains at most one chord, then $G$ is of class 1.

Proof. Suppose that $G$ is a counterexample to our theorem with the minimum number of edges and suppose that $G$ is embedded in the plane. Then $G$ is a 6-critical graph by Lemmas 5 and 6 , and it is 2-connected. By Euler's formula $|V(G)|-|E(G)|+|F(G)|=2$, we have

$$
\sum_{v \in V(G)}(d(v)-4)+\sum_{f \in F(G)}(d(f)-4)=-8<0
$$

We define $c h$ to be the initial charge. Let $\operatorname{ch}(x)=d(x)-4$ for each $x \in V \cup F$. So $\sum_{x \in V \cup F} \operatorname{ch}(x)<0$. In the following, we will reassign a new charge denoted by $\operatorname{ch}^{\prime}(x)$ to each $x \in V \bigcup F$ according to the discharging rules. Since our rules only move charges around, and do not affect the sum. If we can show that $\operatorname{ch}^{\prime}(x) \geq 0$ for each $x \in V \cup F$, then we get an obvious contradiction

$0 \leq \sum_{x \in V \cup F} \operatorname{ch}^{\prime}(x)=\sum_{x \in V \cup F} \operatorname{ch}(x)<0$. which completes our proof.

The discharging rules are defined as follows.

R1: Every $5^{+}$-face $f$ sends $\frac{d(f)-4}{d(f)}$ to each incident vertex.

R2: Every 2-vertex receives 1 from each adjacent vertex.

R3: Every 3 -vertex receives $\frac{1}{3}$ from each adjacent vertex.

R4: Let $f$ be a 3-face $[x, y, z]$ with $d(x) \leq d(y) \leq d(z)$. If $2 \leq d(x) \leq 4$ and $\min \{d(y), d(z)\} \geq 5$, then $f$ receives $\frac{1}{2}$ from $y, \frac{1}{2}$ from $z$; If $d(x)=d(y)=4$ and $d(z)=6$ then $z$ sends 1 to $f$; If $\min \{d(x), d(y), d(z)\} \geq 5$, then $x, y, z$ sends $\frac{1}{3}$ to $f$, respectively.

R5: If a 5-vertex $v$ is adjacent to a 6-vertex $x$ and incident with a $(3,5,6)$-face $[u, v, w]$ such that $u x \notin E(G)$ and $w \neq x$, then $x$ sends $\frac{1}{5}$ to $v$.

Now, let's began to check $c h^{\prime}(x) \geq 0$ for all $x \in V \cup F$. Let $f \in F(G)$. Then $d(f) \geq 3$. If $d(f) \geq 5$, then $c h^{\prime}(f) \geq \operatorname{ch}(f)-(d(f)-4)=0$ by R1. If $d(f)=4$, then $c h^{\prime}(f)=\operatorname{ch}(f)=0$. If $d(f)=3$, then $\operatorname{ch}^{\prime}(f) \geq \operatorname{ch}(f)+\max \left\{2 \times \frac{1}{2}, 1,3 \times \frac{1}{3}\right\}=0$ by $\mathrm{R} 4$. Let $w \in V(G)$. Then $d(w) \geq 2$. If $d(w)=2$, then $c^{\prime}(w)=\operatorname{ch}(w)+2 \times \frac{1}{2}=0$ by R2. If $d(w)=3$, then $w$ is adjacent to three $5^{+}$-vertices by Corollary 2 , and it follows that $\operatorname{ch}^{\prime}(w)=\operatorname{ch}(w)+3 \times \frac{1}{3}=0$ by R3. If $d(w)=4$, then $c h^{\prime}(w)=c h(w)=0$.

Since any 6-cycle of G contains at most one chord, we have the following claim.

Claim 1. Let $f, f^{\prime}, f^{\prime \prime}$ be three faces incident with $w$ such that $f^{\prime}$ is adjacent to $f$ and $f^{\prime \prime}$. If $f$ and $f^{\prime \prime}$ are 3-faces, then $f^{\prime}$ must be a $5^{+}$-face.

Suppose that $d(w)=5$. We have $\operatorname{ch}(w)=1$, $f_{3}(w) \leq 3, \quad \min \{d(u) \mid u \in N(w)\} \geq 3, \quad d_{3}(w) \leq 1 \quad$ and $d_{6}(w) \geq 2$. Let $w_{0}, w_{1}, \cdots, w_{4}$ be neighbors of $w$ and $f_{0}, f_{1}, \cdots, f_{4}$ be faces incident with $w$ such that $f_{i}$ is incident with $w_{i}$ and $w_{i+1}$, for all $i \in\{0,1, \cdots, 4\}$, where $w_{5}=w_{0}$. If all neighbors of $w$ are $5^{+}$-vertices, then $\operatorname{ch}^{\prime}(w) \geq \operatorname{ch}(w)+3 \times \frac{1}{3}=0$ by R4. Suppose that $\min \left\{d\left(w_{i}\right): 0 \leq i \leq 4\right\}=4$. If $f_{3}(w) \leq 2$, then 
$\operatorname{ch}^{\prime}(w) \geq 1-2 \times \frac{1}{2}=0$ by R4; Otherwise, without loss of generality, assume that $f_{4}, f_{0}, f_{2}$ are 3-faces. Then $f_{1}$ and $f_{3}$ are $5^{+}$-faces by Claim 1 . By Lemma 4 , $d_{4}(v)=1$. So $w$ sends at most $\left(2 \times \frac{1}{2}+\frac{1}{3}\right)$ to its adjacent 3-faces. At the same time, $w$ receives at least $2 \times \frac{1}{5}$ from $f_{1}$ and $f_{3}$ by $\mathrm{R} 1$, and it follows that $\operatorname{ch}^{\prime}(w) \geq 1+\frac{2}{5}-\left(2 \times \frac{1}{2}+\frac{1}{3}\right)>0$. Suppose that $d_{3}(w)=1$, without loss of generality, assume that $d\left(w_{1}\right)=3$. Then $d_{6}(w)=4$ by Lemma 1 . If $f_{3}(w) \leq 1$, or $f_{3}(w)=2$ and $w w_{1}$ is not incident with a 3 -face, then $c h^{\prime}(w) \geq 1-\frac{1}{3}-\max \left\{\frac{1}{2}, 2 \times \frac{1}{3}\right\}=0$ by R3 and R4; Otherwise, $f_{3}(w) \geq 2$ and then $w$ is incident with a $5^{+}$face. If $f_{3}(w)=2$, then

$c h^{\prime}(w) \geq 1+\frac{1}{5}-\frac{1}{3}-\left(\frac{1}{2}+\frac{1}{3}\right)>0$; Otherwise, $w$ is incident with two $5^{+}$-faces. If $w w_{1}$ is not incident with a 3face, then $c h^{\prime}(w) \geq 1+2 \times \frac{1}{5}-\frac{1}{3}-3 \times \frac{1}{3}>0$ by R3 and R4;

Otherwise, w receives at least $2 \times \frac{1}{5}$ from its neighbors by R5, and it follows that

$\operatorname{ch}^{\prime}(w) \geq 1+4 \times \frac{1}{5}-\frac{1}{3}-\left(2 \times \frac{1}{2}+\frac{1}{3}\right)>0$.

In the following we check the case that $d(w)=6$. Thus we have $\operatorname{ch}(w)=2, f_{3}(w) \leq 4, d_{2}(w) \leq 1$ and $d_{6}(w) \geq 2$ by Lemma 1 .

Case 1. $w$ sends positive charge to some adjacent 5-vertex $v$ (ref. R5).

Suppose that $v$ is incident with a $(3,5,6)$-face $[u, v, x]$ such that $w u \notin E(G)$ and $w \neq x$ (see R5). Then $x$ may sends $\frac{1}{5}$ to $v$ by $\mathrm{R} 5$. At the same time, $w$ is adjacent to five 6-vertices by Lemma 3, that is,

$d_{6}(w)=5$. Since $f_{3}(w) \leq 4, c h^{\prime}(w)=2-\frac{1}{5}-4 \times \frac{1}{3}>0$.

Case 2. $w$ sends no charge to its adjacent $5^{+}$-vertices.

Let $k=\min \{d(u) \mid u \in N(w)\}$. If $k \geq 5$, then $c^{\prime}(w) \geq 2-4 \times \frac{1}{3}>0$. Suppose that $k=4$. Then $d_{6}(w) \geq 3$ by Lemma 1 and $w$ may be incident with a $(4,4,6)$-face. If $f_{3}(w) \leq 3$, then $c h^{\prime}(w) \geq 2-1-\frac{1}{2}-\frac{1}{3}>0$; Otherwise, $f_{5^{+}}(w) \geq 2$ and it follows that $c h^{\prime}(w) \geq 2+\frac{1}{5}-\max \left\{1+\frac{1}{2}+2 \times \frac{1}{3}, 4 \times \frac{1}{2}\right\}>0$.

Suppose that $k=3$. Then $d_{6}(w) \geq 6-3+1=4$ by Lemma 1. If $d_{5^{+}}(w) \geq 5$, then

$c^{\prime}(w) \geq 2-\frac{1}{3}-2 \times\left(\frac{1}{2}+\frac{1}{3}\right)=0$; Otherwise, $w$ is incident with two 4-vertices $u, v$, then $u$ and $v$ are incident with at most one 3 -face by Lemma 4 since $d(u)+d(v)+d(w) \leq 3+4+6<14$. So $f_{3}(w) \leq 3$ and it follows that $c h^{\prime}(w) \geq 2-2 \times \frac{1}{2}-2 \times \frac{1}{3}>0$ by R3 and R4.

Suppose that $k=2$, that is, $w$ is adjacent to a 2-vertex $v$. Then $d_{6}(w)=5$ by Lemma 1 . If $f_{3}(w)=4$, then $f_{5^{+}}(w)=2$ and it follows that $c h^{\prime}(w) \geq 2+\frac{2}{5}-1-\frac{1}{2}-2 \times \frac{1}{3}>0$; Otherwise, $\operatorname{ch}^{\prime}(w) \geq 2-1-\max \left\{\frac{1}{2}+2 \times \frac{1}{3}-\frac{1}{5}, 3 \times \frac{1}{3}\right\}=0$.

\section{REFERENCES}

[1] S. Fiorini and R. J. Wilson, "Edge-Colorings of Graphs," In: S. Fiorini and R. J. Wilson, Eds., Edge-Colorings of Graphs, Vol. 16, Pitman, London, 1977.

[2] H. Hind and Y. Zhao, "Edge Colorings of Graphs Embedable in a Surface of Low Genus," Discrete Mathematics, Vol. 190, No. 1-3, 1998, pp. 107-114. doi:10.1016/S0012-365X(98)00050-8

[3] L. Y. Miao and J. L. Wu, "Edge-Coloring Critical Graphs with High Degree," Discrete Mathematics, Vol. 257, No. 1, 2002, pp. 169-172. doi:10.1016/S0012-365X(02)00395-3

[4] L. M. Zhang, "Every Planar Graph with Maximum Degree 7 Is of Class 1," Graphs and Combinatorics, Vol. 16, No. 4, 2000, pp. 467-495. doi:10.1007/s003730070009

[5] D. P. Sanders and Y. Zhao, "Planar Graphs of Maximum Degree Seven Are Class 1," Journal of Combinatorial Theory, Series B, Vol. 83, No. 2, 2001, pp. 202-212. doi:10.1006/jctb.2001.2047

[6] P. Lam, J. Liu, W. Shiu and J. Wu, "Some Sufficient Conditions for a Planar Graph to Be of Class 1," Congressus Numerantium, Vol. 136, No. 4, 1999, pp. 201205.

[7] G. F. Zhou, "A Note on Graphs of Class 1," Discrete Mathematics, Vol. 263, No. 1-3, 2003, pp. 339-345. doi:10.1016/S0012-365X(02)00793-8

[8] Y. H. Bu and W. F. Wang, "Some Sufficient Conditions for a Planar Graph of Maximum Degree Six to Be Class 1," Discrete Mathematics, Vol. 306, No. 13, 2006, pp. 1440-1445. doi:10.1016/j.disc.2006.03.032

[9] W. P. Ni, "Edge Colorings of Planar Graphs with $\Delta=6$ without Short Cycles Contain Chords," Journal of Nanjing Normal University, Vol. 34, No. 3, 2011, pp. 19-24 (in Chinese). 